\section{Immunomodulation with microbial vaccines to prevent type 1 diabetes mellitus}

Nikolai Petrovsky

\begin{abstract}
Selected bacteria, viruses, parasites and nonliving, immunologically active microbial substances prevent autoimmune diabetes in animal models. Such agents might also have a protective effect in humans by providing immune stimuli critical during childhood development. The 'hygiene hypothesis' proposes that reduced exposure to environmental stimuli, including microbes, underlies the rising incidence of childhood autoimmune diseases, including type 1 diabetes mellitus (T1DM). This hypothesis is supported by data that highlight the importance of infant exposure to environmental microbes for appropriate development of the immune system, which might explain the observation that administration of microbes or their components inhibits autoimmune disease in animals. This finding raises the possibility of using live, nonpathogenic microbes (for example, probiotics) or microbial components to modulate or 're-educate' the immune system and thereby vaccinate against T1DM. Progress has been assisted by the identification of receptors and pathways through which gut microbes influence development of the immune system. Such mechanistic data have moved a field that was once regarded as being on the scientific fringe to the mainstream, and support increased funding to advance this promising area of research in the hope that it might deliver the long awaited answer of how to safely prevent T1DM.
\end{abstract}

Petrovsky, N. Nat. Rev. Endocrinol. 6, 131-138 (2010); doi:10.1038/nrendo.2009.273

\section{Introduction}

Type 1 diabetes mellitus (T1DM) is an autoimmune disease characterized by selective, T-cell-mediated destruction of pancreatic islet $\beta$ cells. ${ }^{1,2}$ Although nonspecific immunosuppression can somewhat delay the progression of T1DM, the substantial short-term and long-term toxic effects of previously used immunosuppressive agents, such as cyclosporine, azathioprine and cyclophosphamide, has prevented their adoption into clinical practice. ${ }^{3-5} \mathrm{New}$ immunosuppressive agents that might have a reduced toxicity, such as antibodies against lymphocyte antigens (CD3, CD4 and CD20) or the immunosuppressant drugs tacrolimus, sirolimus, and mycophenolate mofetil are currently being tested in preclinical and clinical studies as potential tools to decrease the risk of T1DM. ${ }^{6,7}$ Whether any of these new immunosuppressive therapies will be sufficiently protective and safe to be used routinely for prevention of T1DM in susceptible individuals is not yet known.

A T1DM vaccine, specific for either a $\beta$-cell protein or other relevant antigen, could offer many potential advantages over nonspecific immunosuppression, including greater safety and long-term protection without the need for ongoing treatment. ${ }^{8}$ Ideally, a T1DM vaccine should produce a long-term immune response that specifically blocks autoimmunity but without otherwise interfering with immune function. This goal could be effected via

\section{Competing interests}

The author declares an association with the following company: Vaxine Pty. See the article online for full details of the relationship. various mechanisms, for example, by changing a $\beta$-cell destructive immune response (mediated by T-helper-1 $\left[\mathrm{T}_{\mathrm{H}} 1\right]$ lymphocytes) to a nondestructive response (mediated by $\mathrm{T}_{\mathrm{H}} 2$ lymphocytes); inducing the production of antigen-specific regulatory $\mathrm{T}$ cells; inactivating autoreactive T cells or making them $\beta$-cell-tolerant; preventing interactions between effector cells; or blocking immune-mediated $\beta$-cell-apoptosis.

Previously used approaches include the administration of tissue-specific or antigen-specific vaccine therapies, for example parenteral or mucosal immunization with self-antigens (such as insulin, GAD65, heat shock proteins and various antigenic peptides). ${ }^{9}$ Although positive results have been reported in animal models and in small early phase trials, an antigen-specific vaccine has yet to be proven effective in a large, placebo-controlled, phase 3 clinical trial. A potential problem associated with antigen-specific approaches is that these antigens have been identified through their recognition by autoantibodies, whereas the critical antigens driving $\beta$-cell destruction are T-cell targets, the identity of which remains elusive. Hence, an alternative approach is the use of nonantigen-specific vaccines able to downregulate autoreactive T-cell responses, removing the need to first identify the T-cell autoantigen that drives $\beta$-cell destruction. The earliest clues to the ability of nonantigenspecific vaccine approaches to modulate autoimmune diabetes came from studies into the nonobese diabetic (NOD) mouse model, identified in 1980 by Japanese investigators searching for a cataract-prone mouse. ${ }^{10}$ 


Key points
- Exposure to environmental microbes is critical for development of a healthy
immune system
- The increasing incidence of autoimmune disease may be attributable to
insufficient microbial exposure owing to improved hygiene in the developed
world ("the hygiene hypothesis")
- Enhanced microbial exposure (for example, by ingestion of probiotics) is safe
and has beneficial immunomodulatory effects
- The use of microbial-based vaccines to prevent type 1 diabetes mellitus is a
novel area of research that holds great promise

NOD mice are prone to spontaneous, immunemediated diabetes and are widely used as a surrogate to study the pathogenesis of T1DM and to test the efficacy of candidate therapies. ${ }^{10}$ Although the NOD mouse model is far from perfect and diabetes in these mice is much easier to avert than in humans, it remains the best model of spontaneous $\beta$-cell autoimmunity in which to test potential T1DM therapies. Notably, almost all T1DM therapies that have been taken into clinical trials have first been shown to be effective in the NOD model. The NOD mouse exhibits multiple immune defects even before the onset of diabetes, with a similar pattern of immune defects as is observed in the diabetes-prone BioBreeding (BB) rat ${ }^{11}$ and humans with T1DM. ${ }^{12}$ Thus, immune system defects predate the development of autoimmune diabetes, which points to immune-system development in early childhood as a critical juncture at which the right environmental signals ensure development of a normal healthy immune system with tolerance for self-antigens. Alternatively, in the absence of such signals, a defective immune system develops that is prone to autoimmunity.

This observation is consistent with the hygiene hypothesis, which posits a critical role of microbial organisms in the signals that drive normal immune system development. ${ }^{13}$ According to this hypothesis, a major factor behind the increasing incidence of childhood immune diseases, including T1DM and allergy, in developed countries may be the reduced exposure of children and their immune systems to environmental microbes. ${ }^{14}$ This reduced exposure is proposed to have occurred as a consequence of improved hygiene, including access to purified drinking water and food free from microbial contamination, and reduced infections as a consequence of vaccination and antibiotic therapy. Thus, invoking a potential 'microbial immune-education' model, appropriate microbial exposure in early life (for example, by the organisms delivered in breast-milk that colonize a newborn baby's gut) might provide critical 'educational' signals that drive development of a healthy immune system. Strong support for this model comes from studies that show a critical role for intestinal microbes in the development of the intestinal immune system. ${ }^{15}$ If correct, susceptibility to autoimmunity could then derive from failure to deliver the appropriate microbes that provide these immune developmental signals; for example, from failure to breast feed or from receptor defects that prevent the baby's immune system from appropriately responding to these microbial signals. The fact that the overwhelming majority of microbes that humans are exposed to are commensal bacteria in the large intestine might explain why dietary modifications that change the gut flora might also influence susceptibility to autoimmune disease. ${ }^{16}$

This theory raises the question as to whether microbes or microbial components could be effective as a nonantigenspecific immunotherapy or vaccine to protect against T1DM. ${ }^{14}$ Although-as discussed below-the data on the use of microbial stimuli to prevent autoimmune disease remain preliminary and are mainly derived from animal studies, the idea has potential. If it were to be proven effective, reducing T1DM risk by giving at-risk children a dietary supplement of probiotic organisms or a single course of vaccination would be a most attractive option for any parent.

\section{Methods of vaccination against T1DM}

Several methods of vaccination against T1DM have been reported, each associated with potential advantages and disadvantages (Table 1). The biological mechanisms that underpin the mode of action of these candidate T1DM vaccines vary widely (Table 1 and Figure 1 ).

\section{Microbial infections}

Exposure to infectious agents is an important environmental factor that influences diabetes status in NOD mice: these animals exhibit a high incidence of spontaneous autoimmune diabetes only when housed under specific, pathogen-free conditions. Autoimmune diabetes can be averted in NOD mice and diabetes-prone BB rats by immunization with the widely used vaccine adjuvant Freund's complete adjuvant (FCA), which contains killed mycobacteria in an oil emulsion. ${ }^{17,18}$ Although FCA is a potent immunostimulant, it can induce extremely severe reactions, including skin necrosis, as a result of excessive immunostimulation, and is unsuitable for human use. Bacillus Calmette-Guérin (BCG) is a suspension of a live, attenuated strain of Mycobacterium tuberculosis that is routinely used for childhood vaccination against tuberculosis. Similar to FCA, inoculation of young NOD mice with BCG averted the development of autoimmune diabetes. ${ }^{19}$ However, despite such a protective effect in animal studies and a pilot study in humans, ${ }^{20}$ subsequent larger and better-powered trials of BCG vaccination in individuals with recent-onset T1DM yielded negative results. ${ }^{21}$ The apparent ineffectiveness of BCG for the prevention of T1DM in humans might be explained by its much weaker immunostimulatory effect than FCA. ${ }^{22}$

Infection with a live, attenuated strain of Salmonella typhimurium was shown to prevent autoimmune diabetes in the NOD mouse. ${ }^{23}$ The protective mechanism was suggested to involve altered chemokine expression and priming and trafficking of $\mathrm{T}$ cells in the infected mice, leading to downregulation of autoreactive $\mathrm{T}$ cells. The same group of researchers also showed that infection of NOD mice with the parasitic trematode Schistosoma mansoni prevented diabetes, as did infection with the gastrointestinal helminths Trichinella spiralis or Heligmosomoides polygyrus. ${ }^{24}$ Protection from diabetes in 
Table 1 | Comparison of candidate microbial T1DM vaccines

\begin{tabular}{|c|c|c|c|}
\hline Type of T1DM vaccine & Mechanism of action & Advantages & Disadvantages \\
\hline \multicolumn{4}{|l|}{ Inactivated microbial vaccines } \\
\hline Freunds complete adjuvant & Unknown & Positive animal data & Too toxic for human use \\
\hline Q fever antigen & $\begin{array}{l}\text { Induction of regulatory T-cell response } \\
\text { through IFN- } \gamma \text { and nitric oxide }\end{array}$ & Existing human vaccine & $\begin{array}{l}\text { Negative results in pilot } \\
\text { human trial }\end{array}$ \\
\hline Bacterial DNA with CpG motif & Activation of TLR9 & Human product available & Animal data equivocal \\
\hline $\begin{array}{l}\text { Escherichia coli } \\
\text { lipopolysaccharide }\end{array}$ & Induction of regulatory T-cell response & Characterized compound & Low therapeutic-toxicity margin \\
\hline $\begin{array}{l}\text { Klebsiella pneumoniae } \\
\text { glycoprotein extract }\end{array}$ & Induction of regulatory T-cell response & Nontoxic & Poorly defined bacterial extract \\
\hline $\begin{array}{l}\text { Escherichia coli } \\
\text { extract OM-85 }\end{array}$ & $\begin{array}{l}\text { Induction of TGF- } \beta \text { through TLR } \\
\text { accessory molecule MyD88 }\end{array}$ & $\begin{array}{l}\text { Defined mechanism } \\
\text { of action }\end{array}$ & Poorly defined bacterial extract \\
\hline $\begin{array}{l}\text { Escherichia coli } \\
\text { extract OM-89 }\end{array}$ & $\begin{array}{l}\text { Immune deviation to a noncytotoxic } \\
\mathrm{T}_{\mathrm{H}} 2 \text { response }\end{array}$ & $\begin{array}{l}\text { Defined mechanism } \\
\text { of action }\end{array}$ & Poorly defined bacterial extract \\
\hline Heat-labile enterotoxin & $\begin{array}{l}\text { Induction of regulatory T-cell response } \\
\text { and increased production of IL-10 }\end{array}$ & Characterized compound & $\begin{array}{l}\text { Potential safety issues related } \\
\text { to use of enterotoxin }\end{array}$ \\
\hline \multicolumn{4}{|l|}{ Live pathogens } \\
\hline Schistosoma mansoni & $\begin{array}{l}\text { Immune deviation to a noncytotoxic } \\
\mathrm{T}_{\mathrm{H}} 2 \text { response }\end{array}$ & Positive animal data & Live pathogen \\
\hline Salmonella typhimurium & $\begin{array}{l}\text { Downregulation of autoreactive } \\
T \text { cells through altered chemokine } \\
\text { expression }\end{array}$ & Positive animal data & Live pathogen \\
\hline Trichinella spiralis & $\begin{array}{l}\text { Immune deviation to a noncytotoxic } \\
\mathrm{T}_{H} 2 \text { response }\end{array}$ & Positive animal data & Live pathogen \\
\hline Heligmosomoides polygyrus & $\begin{array}{l}\text { Immune deviation to a noncytotoxic } \\
\mathrm{T}_{\mathrm{H}} 2 \text { response }\end{array}$ & Positive animal data & Live pathogen \\
\hline Lymphotropic virus & Unknown & Positive animal data & Live pathogen \\
\hline BCG & Altered immune-cell trafficking & Existing human vaccine & Equivocal human data \\
\hline \multicolumn{4}{|l|}{ Probiotic organisms } \\
\hline Probiotics & $\begin{array}{l}\text { Downregulation of inflammation } \\
\text { by production of SCFAs that bind } \\
\text { to GPR43 }\end{array}$ & $\begin{array}{l}\text { Safe to use in humans } \\
\text { Oral administration }\end{array}$ & None known \\
\hline VSL\# $3^{\circledR *}$ & $\begin{array}{l}\text { T-cell mediated protection associated } \\
\text { with increased IL-10 }\end{array}$ & $\begin{array}{l}\text { Safe to use in humans } \\
\text { Oral administration }\end{array}$ & None known \\
\hline Lactobacillus casei & $\begin{array}{l}\text { T-cell mediated protection associated } \\
\text { with increased production of IL-4, IL-5, } \\
\text { IL-6 and IL-10 }\end{array}$ & $\begin{array}{l}\text { Safe to use in humans } \\
\text { Oral administration }\end{array}$ & None known \\
\hline Bacteroides fragilis & $\begin{array}{l}\text { Capsular polysaccharide activates } \\
\text { dendritic cells, stimulating lymphoid } \\
\text { organogenesis }\end{array}$ & $\begin{array}{l}\text { Safe to use in humans } \\
\text { Oral administration }\end{array}$ & None known \\
\hline PRODIA study ${ }^{52}$ formulation ${ }^{\ddagger}$ & $\begin{array}{l}\text { General protective effects } \\
\text { of probiotics }\end{array}$ & $\begin{array}{l}\text { Safe to use in humans } \\
\text { Oral administration }\end{array}$ & None known \\
\hline
\end{tabular}

*Comprises Bifidobacterium breve, Bifidobacterium longum, Bifidobacterium infantis, Lactobacillus acidophilus, Lactobacillus plantarum, Lactobacillus paracasei, Lactobacillus bulgaricus and Streptococcus thermophilus (VSL3 Pharmaceuticals, Gaithersburg, MD). ${ }^{\ddagger}$ Comprises Lactobacillus rhamnosus, Bifidobacterium breve and Propionibacterium freudenreichii ssp. Shermani JS. Abbreviations: BCG, Bacillus Calmette-Guérin; GPR43, G-protein coupled receptor 43; IFN- $\gamma$, interferon $\gamma$;

IL, interleukin; SCFAs, short chain fatty acids; T1DM, type 1 diabetes mellitus; TGF- $\beta$, transforming growth factor $\beta ; T_{H} 2$, T-helper-2; TLR, toll-like receptor.

the helminth models was postulated to involve immune deviation towards a $\mathrm{T}_{\mathrm{H}} 2$-response, which in NOD mice has been associated with reduced $\beta$-cell destruction. ${ }^{25}$

Viruses can also prevent diabetes in animal models. Newborn and adult NOD mice infected with lymphotropic virus were protected against diabetes, suggesting that viruses and/or host factors induced by viral infection might have potential for prevention of T1DM. ${ }^{26,27}$ Reducing the environmental exposure of diabetesprone rats to virus by cesarean delivery accelerates and increases the frequency of diabetes within the colony, again consistent with a protective effect of viral infection against autoimmune diabetes. ${ }^{28}$

Despite the abundance of data on bacterial, trematode, helminithic and viral protection against autoimmune diabetes in animal studies, no human infection or colonization has yet been shown to protect against T1DM. Substantial risks could be associated with any use of live pathogens, such as BCG, Salmonella typhimurium, Schistosoma mansoni or viruses, to prevent T1DM in infants. However, as discussed below, such safety barriers would be alleviated if it were possible to develop T1DM 


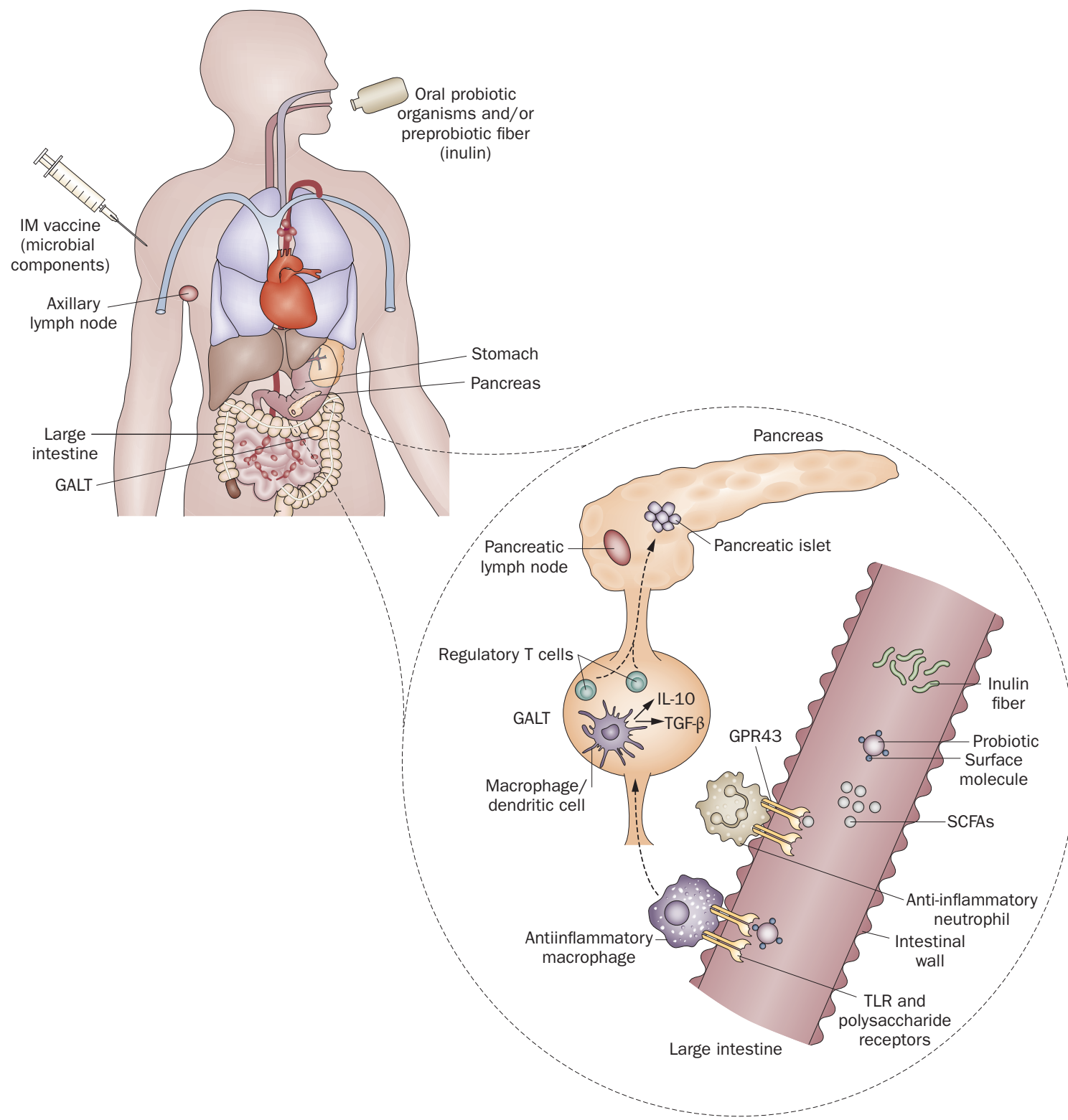

Figure 1 | Mechanism of action of microbial vaccines against T1DM. Inactivated or subunit microbial vaccines administered $\mathrm{IM}$ are bound and phagocytosed by muscle macrophages and dendritic cells. These cells become activated, start to express regulatory cytokines (IL-10 and TGF- $\beta$ ) and migrate systemically via the axillary lymph node to the pancreatic lymph nodes where they stimulate regulatory T cells, which in turn suppress $\beta$-cell destruction mediated by autoreactive T cells. Orally ingested live probiotic vaccines mixed with preprobiotic inulin fiber enter the large intestine where the probiotic organisms ferment the inulin to produce SCFAs. The SCFAs bind to GPR43 on immune cells that line the intestinal wall, delivering an anti-inflammatory signal within the GALT. At the same time, polysaccharides, glycolipids and proteins in the probiotic cell wall bind to other immune receptors, such as TLR, on intestinal macrophages, inducing them to adopt an antiinflammatory phenotype and migrate to the pancreatic lymph nodes where they induce regulatory T cells, which in turn inhibit autoreactive T cells responsible for $\beta$-cell destruction. Abbreviations: GALT, gut-associated lymphoid tissue; GPR43, G-protein coupled receptor 43; IL-10, interleukin 10; IM, intramuscular; SCFAs, short-chain fatty acids; T1DM, type 1 diabetes mellitus; TGF- $\beta$, transforming growth factor $\beta$; TLR, toll-like receptor.

therapies that mimic the protective effects of live pathogens, but which comprise the microbial components mediating these beneficial effects rather than the whole living organism.

\section{Inactivated microbial vaccines}

As mentioned above, the risks associated with the use of live organisms could be avoided by using inactivated or subunit vaccine formulations. Q fever complementfixing antigen (QFA) derived from formalin-inactivated Coxiella burnetii is the basis of an Australian Q fever vaccine that has been in use for several decades. ${ }^{29-31}$ $\mathrm{Q}$ fever infections are caused by Coxiella burnetii, a rickettsia-like organism with worldwide distribution. Coxiella burnetii is a common pathogen of wild animals and domesticated livestock; human infection occurs 
by inhalation of aerosolized matter from dead, infected animals or by drinking infected, raw milk. ${ }^{32,33}$ Vaccination with QFA produces a long-term, protective immune response against $\mathrm{Q}$ fever and induces a potent, nonspecific suppression of unrelated infectious organisms, including malarial parasites, ${ }^{34}$ bacteria and viruses. ${ }^{35}$ This nonspecific effect of QFA has been attributed to increased production of cytokines, such as tumor necrosis factor (TNF), interferon $\gamma$ (IFN- $\gamma$ ) and nitric oxide (NO), which are associated with cell-mediated immune responses, ${ }^{34}$ and are similar to the immune responses evoked by FCA and BCG, which suggests that QFA might also share their diabetes-protective effects.

Indeed, QFA administered subcutaneously to young NOD mice provided robust protection against diabetes. ${ }^{36}$ QFA therapy also preserved islet $\beta$-cell mass in pancreas sections of 300-day-old NOD mice better than did BCG or FCA and prevented recurrent autoimmune destruction of syngeneic islets grafted into diabetic NOD mice. ${ }^{36} \mathrm{QFA}$ is a potent inducer of cytokines, particularly IFN- $\gamma \cdot{ }^{30} \mathrm{QFA}$ protection against diabetes was lost in IFN- $\gamma$-deficient NOD mice, indicating an essential role of IFN- $\gamma$ in diabetes protection. ${ }^{36} \mathrm{As} \mathrm{IFN}-\gamma$ is an important inducer of NO production, the protective effect of QFA might be regulated via a NO-mediated mechanism. This effect would be consistent with studies in other models of autoimmune disease, for example experimental autoimmune encephalomyelitis, where NO protected against autoimmune disease. ${ }^{37-39} \mathrm{~A}$ pilot clinical trial of QFA in patients with recent-onset T1DM did not show remarkable effects, indicating the need for further refinement of this approach.

\section{Subunit microbial vaccines}

Although use of inactivated organisms like QFA is safer than the use of live pathogens, there remains a risk of adverse reactions. An even better approach, therefore, would be to identify and extract the protective microbial component for use as a subunit vaccine. Such a strategy is feasible as purified bacterial components, including a lipopolysaccharide from Escherichia coli and glycoprotein extracts from Klebsiella pneumoniae, reduced diabetes when fed to NOD mice. ${ }^{40}$ Lymphocytes taken from these orally immunized mice had a reduced capacity to induce $\beta$-cell destruction and diabetes when transplanted into healthy recipient mice, consistent with a role for these microbial components in inducing a regulatory T-cell response. Diabetes in NOD mice was also successfully prevented by oral administration of Escherichia coli-derived extract OM-89, in combination with insulin. ${ }^{41}$ Potentiation of the effect of oral insulin by OM- 89 was associated with an altered $\mathrm{T}_{\mathrm{H}} 2$-mediated immune response, characterized by upregulation of interleukin 4 (IL-4) producing $\mathrm{T}_{\mathrm{H}} 2$ cells in infiltrated islets and downregulation of inducible NO synthase. ${ }^{41}$

Toll-like receptors (TLRs) expressed on immune cells have a critical role in immune recognition of microbial components, including bacterial and viral DNA, RNA, proteins and lipoproteins. Host-cell TLRs may be an important link between microbial exposure and protection against autoimmunity. Interestingly, CpG-containing DNA that specifically binds and activates TLR9 is a major immunostimulatory component of BCG and might contribute to the ability of BCG to inhibit diabetes in NOD mice. Nonprotein-coding DNA plasmids containing CpG motifs decreased insulitis when injected into NOD mice, consistent with an inhibitory effect of bacterial DNA on autoimmune diabetes. ${ }^{42}$ However, other studies of vaccination of NOD mice with bacterial CpG-containing DNA found no protective effect on diabetes, suggesting that as yet uncharacterized features of some DNA molecules might be critical for diabetes prevention. ${ }^{43}$

An alternative subunit approach involved oral or intraperitoneal administration of the bacterial extract OM-85, which delayed or completely prevented, respectively, diabetes in NOD mice. ${ }^{44}$ Optimal protection was observed when treatment was started at 3-6 weeks of age, although effects were still observed when started as late as 10 weeks of age. The therapeutic effect of OM-85 depended upon transforming growth factor $\beta$ (TGF- $\beta$ ) and the activity of natural killer T cells. OM-85 is a complex mixture of bacterial components and the active constituent has yet to be identified. Lipopolysaccharides were excluded as the active component, but one or more other TLR-agonists might be involved, as induction of TGF- $\beta$ by OM- 85 is dependent on the TLR-accessory molecule, MyD88. ${ }^{44}$

Other successful subunit vaccine approaches not involving TLRs include intranasal treatment with the B-subunit of a Escherichia coli-derived protein, heat-labile enterotoxin, which also prevented diabetes in NOD mice. ${ }^{45}$ This effect was associated with a reduction in the number of macrophages, $\mathrm{CD} 4^{+} \mathrm{T}$ cells, $\mathrm{B}$ cells, and major histocompatiblity complex (MHC) II-bearing cells in the pancreatic islets. Treated mice had increased numbers of IL-10-producing cells in the pancreas, and a decreased cytokine production by $\mathrm{T}_{\mathrm{H}} 1$ cells and $\mathrm{T}_{\mathrm{H}} 2$ cells in the pancreatic lymph node. Protection against diabetes could be transferred by injecting $\mathrm{CD} 4^{+} \mathrm{T}$ cells from treated mice to untreated recipients. ${ }^{45}$ However, FDA approval for the use of such toxins in T1DM prevention might be problematic, given that a similar enterotoxin caused facial palsy in a human trial of nasal influenza vaccine. ${ }^{46}$

\section{Probiotic organisms}

Commensal microorganisms that confer a health benefit on the host (probiotics) might have an overlooked role in T1DM prevention. The large intestine contains many families of commensal bacteria and yeasts that have important health-promoting roles. For example, germ-free animals have impaired immune system development that can be corrected by gut colonization with the ubiquitous microorganism Bacteroides fragilis. ${ }^{15}$ Interestingly, susceptibility of NOD mice to autoimmune diabetes is dependent on their being raised in a germ-free environment, ${ }^{10}$ consistent with a protective effect of microbial colonization.

Direct evidence that probiotics inhibit diabetes pathogenesis is provided by VSL \#3 ${ }^{\circledR}$ (VSL3 Pharmaceuticals, Gaithersburg, MD), a probiotic that consists of eight different strains of lactic-acid producing bacteria (Bifidobacterium breve, Bifidobacterium longum, Bifidobacterium infantis, Lactobacillus acidophilus, Lactobacillus plantarum, 
Lactobacillus paracasei, Lactobacillus bulgaricus, and Streptococcus thermophilus). When given orally to NOD mice, VSL $\# 3^{\circledR}$ prevented diabetes, with increased production of the regulatory cytokine IL-10 seen in Peyer's patches, the spleen and the pancreas of these mice. ${ }^{47}$ The protective effect of VSL\# $3^{\circledR}$ was transferable to irradiated mice receiving diabetogenic $\mathrm{T}$ cells by injection of splenocytes from VSL $\# 3^{\circledR}$-treated animals. ${ }^{47}$ Although no experience in humans with T1DM has been reported, VSL $\# 3^{\circledR}$ has been shown to be safe and to reduce colon inflammation and induce remission in children with ulcerative colitis, making it a promising candidate for T1DM studies. ${ }^{48}$

Protective effects against autoimmune diabetes have also been observed using the probiotic, Lactobacillus casei. Young NOD mice treated with orally administered Lactobacillus casei showed preservation of $\beta$ cells with an increased ratio of CD $45 \mathrm{R}^{+} \mathrm{B}$ cells and $\mathrm{CD} 8^{+} \mathrm{T}$ cells in the spleen, decreased production of IFN- $\gamma$ and increased production of IL-4, IL-5, IL- 6 and IL- $10 .{ }^{49}$ The antiinflammatory effects of Lactobacillus casei were also seen in the KKAy mouse model of type 2 diabetes mellitus where oral administration inhibited the production of inflammatory cytokines by T cells..$^{50}$ Alloxan is a toxin that generates reactive oxygen species and induces $\beta$-cell death which mimics that seen in patients with T1DM. ${ }^{51}$ Administration of Lactobacillus casei protected against alloxan-induced diabetes in 7-week-old BALB/c mice, consistent with a protective effect of this probiotic on inflammation-induced $\beta$-cell death. ${ }^{51}$

Probiotics are an attractive choice for a T1DM intervention because of their longstanding safety record and widespread public acceptance. The PRODIA study ${ }^{52}$ is currently testing whether oral administration of a probiotic mixture of Lactobacillus rhamnosus, Bifidobacterium breve and Propionibacterium freudenreichii ssp. Shermani JS during the first 6 months of life reduces the development of $\beta$-cell autoantibodies in children with an increased genetic risk for T1DM. ${ }^{52}$ If the results of this trial are positive, interest in the use of probiotics to regulate immune function in children at risk of autoimmune disease is likely to increase substantially.

\section{Mechanisms of microbial protection}

A number of hypotheses have been put forward to explain how microbes and microbial components protect against autoimmune disease. One theory suggests that microbial stimuli shift a proinflammatory $\mathrm{T}_{\mathrm{H}} 1$ response to a noninflammatory $\mathrm{T}_{\mathrm{H}} 2$ response, characterized by production of regulatory cytokines such as IL- 4 and IL-10. ${ }^{41}$ An alternative theory proposes that microbial stimuli induce $\mathrm{CD}^{+}{ }^{+}$regulatory T-cells. ${ }^{53}$ Alternatively, microbial stimuli change immune-cell trafficking and thereby divert autoreactive $\mathrm{T}$ cells away from the site of autoimmune destruction. ${ }^{54,55}$ Thus, administration of FCA to NOD mice reduced the number of infiltrating dendritic cells and T cells and suppressed CCL21 and CD31 expression on the endothelial lining of lymphatic vessels. ${ }^{55}$ Similarly, immunization with Salmonella typhimurium was shown to be mediated by an effect on dendritic cells which altered trafficking of autoreactive T cells and prevented development of diabetes. ${ }^{56,57}$

Most-if not all-of the effects of microbes and microbial components that influence the development of autoimmune diabetes can be tracked back to activation of monocytes or dendritic cells by cell-surface, innate immune receptors, such as TLR, that recognize specific microbial compounds. For example, the immunomodulatory activity of Bacteroides fragilis on the gut immune system was shown to be mediated by a specific bacterial polysaccharide that, when ingested by intestinal dendritic cells, activated $\mathrm{CD} 4^{+} \mathrm{T}$ cells, and stimulated lymphoid organogenesis. ${ }^{15}$ The critical role of the bacterial polysaccharide was confirmed by showing that a Bacteroides fragilis strain that expressed a mutant polysaccharide did not restore gut immune function. ${ }^{15}$

NOD mice genetically lacking the MyD88 adaptor protein critical for TLR signaling were protected against autoimmune diabetes when housed under specific pathogen-free conditions. ${ }^{58}$ The protective effect of the block to TLR signaling was dependent on commensal gut microbes: germ-free and MyD88-negative NOD mice continued to develop autoimmune diabetes, whereas probiotic colonization of such mice with a defined microbial consortium restored protection. ${ }^{58}$ As MyD88 is the major signaling molecule in the TLR pathway in monocytes and dendritic cells, the above findings suggest that these immune cells have an important role in immune education by microbial stimuli. A gluten-free diet has also been shown to reduce autoimmune diabetes in NOD mice at the same time as substantially changing the bacterial flora in their intestines. ${ }^{16}$ Surprisingly, a gluten-rich diet also reduced the incidence of diabetes in NOD mice, ${ }^{59}$ consistent with the idea that dietary manipulations achieve beneficial outcomes through a common effect on modulating the intestinal flora. Some exciting data showed that short-chain fatty acids produced by gut commensal bacteria downregulate inflammatory responses by stimulating the chemoattractant receptor GPR43 on neutrophils providing further mechanistic data on how microbial stimuli act to suppress autoimmune disease. ${ }^{60}$

Taken together, the above data suggest that microbial vaccines protect against autoimmune diabetes through their ability to activate and modulate host immune cells, driving normal development of the immune system. Microbial compounds including lipopolysaccharides, polysaccharides, RNA or DNA bind to host cell receptors, such as TLRs, resulting in activation of monocytes and dendritic cells. These activated immune cells interact in turn with antigen-specific $\mathrm{T}$ and $\mathrm{B}$ cells, leading to alterations in cell trafficking, cytokine expression and regulatory function that together act to suppress autoreactive T cells. Such microbial effects on immune function are not unique to pathogens, such as mycobacteria, but are also mediated by commensal organisms (probiotics) resident in the human intestine. This finding presents a unique opportunity to modulate and prevent autoimmune disease by regulating the gut environment, either by administering health-promoting probiotic organisms or by appropriately altering infant diets, for example, by encouraging breast 
feeding or supplementing the diet with soluble fibers such as inulin that stimulate probiotic growth..$^{58}$ Studies of dietary interventions, such as infant avoidance of exposure to cow's milk to prevent T1DM in children, are already underway.

\section{Conclusions}

Clinically proven strategies for long-term prevention of T1DM remain elusive. Whereas modest benefits have been achieved in clinical trials with various immunosuppressive therapies, short-term and long-term toxicity remain a major concern. Extensive animal data supports the idea that genetic and acquired immune defects that underlie susceptibility to autoimmune diabetes might be corrected by early-life exposure to specific microbes or microbial components. Activation of immune receptors (for example, TLRs, GPR43 and polysaccharide receptors) by microbes and microbial components is critical to normal immune system development and provides important antiinflammatory signals within the intestinal environment. With increasing hygiene, these microbial stimuli become insufficient to drive maturation of the immune system and suppression of inflammation, particularly in genetically susceptible individuals, resulting in aberrant immune system development and autoimmune disease. In such individuals, inadequate microbial immune stimulation could be supplemented either by intramuscular immunization with purified microbial components or by dietary manipulation with probiotics and/or a preprobiotic fiber such as inulin. Although the use of microbes to manipulate the human immune system remains in its infancy, the safety and utility of this approach when compared with other proposed remedies such as immunosuppression, warrants major investment by funding bodies. The fact that two key papers on this topic have recently been published in the high-ranking journal Nature ${ }^{58,60}$ should help dispel doubts as to the scientific credibility of such microbial therapy approaches.

Review criteria
Papers for this Review were identified in PubMed, Medline
and Google using the keyword "diabetes mellitus"
combined with one or more of the following terms:
"immunotherapy", "microbe", "vaccine", "probiotic", and
"pathogen." In addition, a search of references cited in
published reviews on the topic of immunotherapy for type 1
diabetes mellitus was performed, with a focus on articles
published between January 1999 and November 2009.

1. Bottazzo, G. F. et al. In situ characterization of autoimmune phenomena and expression of HLA molecules in the pancreas in diabetic insulitis. N. Engl. J. Med. 313, 353-360 (1985).

2. Foulis, A. K., Liddle, C. N., Farquharson, M. A., Richmond, J. A. \& Weir, R. S. The histopathology of the pancreas in type 1 (insulin-dependent) diabetes mellitus: a 25-year review of deaths in patients under 20 years of age in the United Kingdom. Diabetologia 29, 267-274 (1986).

3. Assan, R. et al. Metabolic and immunological effects of cyclosporin in recently diagnosed type 1 diabetes mellitus. Lancet 1, 67-71 (1985).

4. De Filippo, G., Carel, J. C., Boitard, C. \& Bougnères, P. F. Long-term results of early cyclosporin therapy in juvenile IDDM. Diabetes 45, 101-104 (1996).

5. Stiller, C. R. et al. Effects of cyclosporine immunosuppression in insulin-dependent diabetes mellitus of recent onset. Science $\mathbf{2 2 3}$, 1362-1367 (1984).

6. Rewers, M. \& Gottlieb, P. Immunotherapy for the prevention and treatment of type 1 diabetes: human trials and a look into the future. Diabetes Care 32, 1769-1782 (2009).

7. Bresson, D. \& von Herrath, M. Immunotherapy for the prevention and treatment of type 1 diabetes: optimizing the path from bench to bedside. Diabetes Care 32, 1753-1768 (2009).

8. Petrovsky, N., Silva, D. \& Schatz, D. A. Prospects for the prevention and reversal of type 1 diabetes mellitus. Drugs 62, 2617-2635 (2002).

9. Petrovsky, N., Silva, D. \& Schatz, D. A. Vaccine therapies for the prevention of type 1 diabetes mellitus. Paediatr. Drugs 5, 575-582 (2003).

10. Makino, S. et al. Breeding of a non-obese, diabetic strain of mice. Jikken Dobutsu 29, 1-13 (1980).

11. Crisá, L., Mordes, J. P. \& Rossini, A. A. Autoimmune diabetes mellitus in the BB rat. Diabetes Metab. Rev. 8, 4-37 (1992).

12. Kühtreiber, W. M., Hayashi, T., Dale, E. A. \& Faustman, D. L. Central role of defective apoptosis in autoimmunity. J. Mol. Endocrinol. 31, 373-399 (2003).
13. Bach, J. F. Infections and autoimmune diseases. J. Autoimmun. 25 (Suppl.), 74-80 (2005).

14. Kukreja, A. \& Maclaren, N. K. NKT cells and type-1 diabetes and the "hygiene hypothesis" to explain the rising incidence rates. Diabetes Technol. Ther. 4, 323-333 (2002).

15. Mazmanian, S. K., Liu, C. H., Tzianabos, A. O. \& Kasper, D. L. An immunomodulatory molecule of symbiotic bacteria directs maturation of the host immune system. Cell 122, 107-118 (2005).

16. Hansen, A. K. et al. Diabetes preventive glutenfree diet decreases the number of caecal bacteria in non-obese diabetic mice. Diabetes Metab. Res. Rev. 22, 220-225 (2006).

17. Sadelain, M. W., Qin, H. Y., Lauzon, J. \& Singh, B. Prevention of type I diabetes in NOD mice by adjuvant immunotherapy. Diabetes $39,583-589$ (1990).

18. Sadelain, M. W. et al. Prevention of diabetes in the BB rat by early immunotherapy using Freund's adjuvant. J. Autoimmun. 3, 671-680 (1990).

19. Harada, M., Kishimoto, Y. \& Makino, S. Prevention of overt diabetes and insulitis in NOD mice by a single BCG vaccination. Diabetes Res. Clin. Pract. 8, 85-89 (1990).

20. Shehadeh, N. et al. Effect of adjuvant therapy on development of diabetes in mouse and man. Lancet 343, 706-707 (1994).

21. Elliott, J. F., Marlin, K. L. \& Couch, R. M. Effect of bacille Calmette-Guérin vaccination on C-peptide secretion in children newly diagnosed with IDDM. Diabetes Care 21, 1691-1693 (1998).

22. Gazda, L. S., Baxter, A. G. \& Lafferty, K. J. Regulation of autoimmune diabetes: characteristics of non-islet-antigen specific therapies. Immunol. Cell Biol. 74, 401-407 (1996).

23. Zaccone, P., Fehervari, Z., Phillips, J. M., Dunne, D. W. \& Cooke, A. Parasitic worms and inflammatory diseases. Parasite Immunol. 28, 515-523 (2006).

24. Cooke, A. et al. Infection with Schistosoma mansoni prevents insulin dependent diabetes mellitus in non-obese diabetic mice. Parasite Immunol. 21, 169-176 (1999).

25. Saunders, K. A., Raine, T., Cooke, A. \& Lawrence, C. E. Inhibition of autoimmune type 1 diabetes by gastrointestinal helminth infection. Infect. Immun. 75, 397-407 (2007).

26. Oldstone, M. B. Prevention of type I diabetes in nonobese diabetic mice by virus infection. Science 239, 500-502 (1988).

27. Oldstone, M. B. Viruses as therapeutic agents. I. Treatment of nonobese insulin-dependent diabetes mice with virus prevents insulindependent diabetes mellitus while maintaining general immune competence. J. Exp. Med. 171, 2077-2089 (1990).

28. Like, A. A., Guberski, D. L. \& Butler, L. Influence of environmental viral agents on frequency and tempo of diabetes mellitus in BB/Wor rats. Diabetes 40, 259-262 (1991).

29. Heggers, J. P., Mallavia, L. P. \& Hinrichs, D. J. The cellular immune response to antigens of Coxiella burneti. Can. J. Microbiol. 20, 657-662 (1974).

30. Izzo, A. A. \& Marmion, B. P. Variation in interferongamma responses to Coxiella burnetii antigens with lymphocytes from vaccinated or naturally infected subjects. Clin. Exp. Immunol. 94, 507-515 (1993).

31. Jerrells, T. R., Mallavia, L. P. \& Hinrichs, D. J. Detection of long-term cellular immunity to Coxiella burneti as assayed by lymphocyte transformation. Infect. Immun. 11, 280-286 (1975).

32. Aitken, I. D. et al. Q fever in Europe: current aspects of aetiology, epidemiology, human infection, diagnosis and therapy. Infection 15, 323-327 (1987).

33. Sawyer, L. A., Fishbein, D. B. \& McDade, J. E. Q fever: current concepts. Rev. Infect. Dis. 9, 935-946 (1987).

34. Clark, I. A. Resistance to Babesia spp. and Plasmodium sp. in mice pretreated with an extract of Coxiella burnetii. Infect. Immun. 24, 319-325 (1979). 
35. Waag, D. M., Kende, M., Damrow, T. A. Wood, O. L. \& Williams, J. C. Injection of inactivated phase I Coxiella burnetii increases nonspecific resistance to infection and stimulates lymphokine production in mice. Ann. NY Acad. Sci. 590, 203-214 (1990).

36. Silva, D. G., Charlton, B., Cowden, W. \& Petrovsky, N. Prevention of autoimmune diabetes through immunostimulation with $\mathrm{Q}$ fever complement-fixing antigen. Ann. NY Acad. Sci. 1005, 423-430 (2003).

37. Cowden, W. B., Cullen, F. A., Staykova, M. A. \& Willenborg, D. O. Nitric oxide is a potential downregulating molecule in autoimmune disease: inhibition of nitric oxide production renders PVG rats highly susceptible to EAE. J. Neuroimmunol. 88, 1-8 (1998).

38. Kelly, C. J. \& Gold, D. P. Nitric oxide in interstitial nephritis and other autoimmune diseases. Semin. Nephrol. 19, 288-295 (1999).

39. Staykova, M. A., Cowden, W. \& Willenborg, D. O. Macrophages and nitric oxide as the possible cellular and molecular basis for strain and gender differences in susceptibility to autoimmune central nervous system inflammation. Immunol. Cell Biol. 80, 188-197 (2002).

40. Saï, P. \& Rivereau, A. S. Prevention of diabetes in the nonobese diabetic mouse by oral immunological treatments. Comparative efficiency of human insulin and two bacterial antigens, lipopolysacharide from Escherichia coli and glycoprotein extract from Klebsiella pneumoniae. Diabetes Metab. 22, 341-348 (1996).

41. Hartmann, B., Bellmann, K., Ghiea, I., Kleemann, R. \& Kolb, H. Oral insulin for diabetes prevention in NOD mice: potentiation by enhancing Th2 cytokine expression in the gut through bacterial adjuvant. Diabetologia 40, 902-909 (1997)

42. Quintana, F. J., Rotem, A., Carmi, P. \& Cohen, I. R. Vaccination with empty plasmid DNA or $\mathrm{CpG}$ oligonucleotide inhibits diabetes in nonobese diabetic mice: modulation of spontaneous 60-kDa heat shock protein autoimmunity. J. Immunol. 165, 6148-6155 (2000).

43. Lee, B. J. et al. Limited effect of CpG ODN in preventing type 1 diabetes in NOD mice. Yonsei Med. J. 46, 341-346 (2005).

44. Alyanakian, M. A. et al. Transforming growth factor-beta and natural killer T-cells are involved in the protective effect of a bacterial extract on type 1 diabetes. Diabetes 55, 179-185 (2006).

45. Ola, T. O. \& Williams, N. A. Protection of nonobese diabetic mice from autoimmune diabetes by Escherichia coli heat-labile enterotoxin B subunit. Immunology 117, 262-270 (2006).

46. Lewis, D. J. et al. Transient facial nerve paralysis (Bell's palsy) following intranasal delivery of a genetically detoxified mutant of Escherichia coli heat labile toxin. PLoS One 4, e6999 (2009).

47. Calcinaro, F. et al. Oral probiotic administration induces interleukin-10 production and prevents spontaneous autoimmune diabetes in the nonobese diabetic mouse. Diabetologia 48 , 1565-1575 (2005).

48. Miele, E. et al. Effect of a probiotic preparation (VSL\#3) on induction and maintenance of remission in children with ulcerative colitis. Am. J. Gastroenterol. 104, 437-443 (2009).

49. Matsuzaki, T. et al. Prevention of onset in an insulin-dependent diabetes mellitus model, NOD mice, by oral feeding of Lactobacillus casei. APMIS 105, 643-649 (1997).

50. Matsuzaki, T., Yamazaki, R., Hashimoto, S. \& Yokokura, T. Antidiabetic effects of an oral administration of Lactobacillus casei in a non-insulin-dependent diabetes mellitus (NIDDM) model using KK-Ay mice. Endocr. J. 44, 357-365 (1997).

51. Matsuzaki, T. et al. Effect of oral administration of Lactobacillus casei on alloxan-induced diabetes in mice. APMIS 105, 637-642 (1997).

52. Ljungberg, M., Korpela, R., Ilonen, J., Ludvigsson, J. \& Vaarala, O. Probiotics for the prevention of beta cell autoimmunity in children at genetic risk of type 1 diabetes--the PRODIA study. Ann. NY Acad. Sci. 1079, 360-364 (2006)

53. Luross, J. A., Heaton, T., Hirst, T. R., Day, M. J. \& Williams, N. A. Escherichia coli heat-labile enterotoxin B subunit prevents autoimmune arthritis through induction of regulatory CD4+ T cells. Arthritis Rheum. 46, 1671-1682 (2002).

54. Sewell, D. L. et al. Infection with Mycobacterium bovis BCG diverts traffic of myelin oligodendroglial glycoprotein autoantigenspecific T cells away from the central nervous system and ameliorates experimental autoimmune encephalomyelitis. Clin. Diagn. Lab. Immunol. 10, 564-572 (2003).

55. Qu, P. Ji, R. C., Shimoda, H., Miura, M. \& Kato, S. Study on pancreatic lymphatics in nonobese diabetic mouse with prevention of insulitis and diabetes by adjuvant immunotherapy. Anat. Rec. A Discov. Mol. Cell Evol. Biol. 281, 1326-1336 (2004).

56. Raine, T., Zaccone, P., Mastroeni, P. \& Cooke, A. Salmonella typhimurium infection in nonobese diabetic mice generates immunomodulatory dendritic cells able to prevent type 1 diabetes J. Immunol. 177, 2224-2233 (2006).

57. Zaccone, P. et al. Salmonella typhimurium infection halts development of type 1 diabetes in NOD mice. Eur. J. Immunol. 34, 3246-3256 (2004).

58. Wen, L. et al. Innate immunity and intestinal microbiota in the development of type 1 diabetes. Nature 455, 1109-1113 (2008).

59. Funda, D. P., Kaas, A., Tlaskalová-Hogenová, H. \& Buschard, K. Gluten-free but also glutenenriched (gluten+) diet prevent diabetes in NOD mice; the gluten enigma in type 1 diabetes. Diabetes Metab. Res. Rev. 24, 59-63 (2008).

60. Maslowski, K. M. et al. Regulation of inflammatory responses by gut microbiota and chemoattractant receptor GPR43. Nature 461 1282-1286 (2009). 